\section{Melhoria da qualidade das informações sobre tuberculose a partir do relacionamento entre bases de dados}

\author{
Improved quality of tuberculosis data using \\ record linkage
}

Mejora en la calidad de la información sobre la tuberculosis, de acuerdo con la relación entre bases de datos

\author{
1 Programa de Pós-graduação \\ em Medicina Tropical. \\ Universidade de Brasília, \\ Brasília, Brasil. \\ 2 Instituto de Estudos em \\ Saúde Coletiva, Universidade \\ Federal do Rio de Janeiro, Rio \\ de Janeiro, Brasil. \\ Correspondência \\ P. Bartholomay \\ SQSW 304 bloco C, apto. 205, \\ Brasília, $D F$ \\ 70673-403, Brasil. \\ patibartho@yahoo.com.br
}

\begin{abstract}
The aim of this study was to improve data quality on tuberculosis (TB) after record linkage and outcome correction through probabilistic linkage between the Information System for Notifiable Diseases (SINAN) and the Mortality Information System (SIM). Record linkage was conducted between SINAN records for Brazil in 2008 and 2009 in order to exclude notifications not removed by routine SINAN procedures performed by States and Municipalities. The databases were constructed according to the case outcomes. Linkage between SINAN and SIM used the database resulting from record linkage and SIM data that mentioned TB as the underlying or associated cause from 2008 to 2010 in Brazil. Record linkage decreased the percentage of new cases closed with patient transfer as the outcome, ranging from $34.8 \%$ in 2008 to $35.5 \%$ in 2009. After linkage between SINAN and SIM, the percentage of TB deaths among new cases increased, varying around 15\%. The results highlight the need for attention to data quality for TB treatment outcomes in SINAN.
\end{abstract}

Tuberculosis; Information Systems; Cause of Death
Patricia Bartholomay 1

Gisele Pinto de Oliveira 2

Rejane Sobrino Pinheiro 2

Ana Maria Nogales Vasconcelos 1

\section{Resumo}

O objetivo deste trabalho foi verificar a melhoria da qualidade das informações sobre a tuberculose (TB), após a vinculação de registros e a correção do encerramento por meio do linkage probabilístico do Sistema de Informações de Agravos de Notificação (SINAN) com o Sistema de Informações sobre Mortalidade (SIM). Para a vinculação de registros foi realizado o linkage entre os registros do SINAN do Brasil, anos 2008 e 2009, com o objetivo de excluir as notificações não removidas pelas rotinas do SINAN realizadas por estados e municípios. As bases de dados foram construídas de acordo com o desfecho dos casos. Para o linkage entre SINAN e SIM foi utilizada a base de dados que resultou da vinculação de registros e os registros do SIM que mencionaram TB como causa básica ou associada, entre 2008 e 2010, no Brasil. A vinculação de registros diminuiu o percentual de casos novos com encerramento por transferência, com variação de 34,8\% em 2008 e 35,5\% em 2009. Após o linkage entre SINAN e SIM, o percentual de óbito por TB aumentou, com variação em torno de $15 \%$. Os resultados descrevem uma situação de alerta no que se refere à qualidade dos dados de desfecho de tratamento de TB no SINAN.

Tuberculose; Sistemas de Informação; Causas de Morte 


\section{Introdução}

O Brasil é um dos 22 países priorizados pela Organização Mundial da Saúde (OMS) que concentra $82 \%$ da carga mundial de tuberculose (TB). Em 2011, foram notificados 73.778 casos novos, correspondendo a um coeficiente de incidência de 38,4/100 mil habitantes, e morreram 4.563 pessoas pela doença, levando a uma mortalidade de 2,4/100 mil habitantes. Ocupa a 17a posição em relação ao número de casos e a 111a posição em relação ao coeficiente de incidência, entre os países do mundo. Devido a essa magnitude, desde 2003, o Ministério da Saúde considera a TB uma prioridade de governo e reúne esforços com estados, municípios e sociedade civil para alterar essa realidade 1,2 .

A TB é considerada uma doença de notificação compulsória há quase 40 anos (1976) e compõe o Sistema de Informações de Agravos de Notificação (SINAN) desde 1990, ano da implantação do mesmo 3,4. A alimentação regular da base de dados nacional pelos municípios, estados e Distrito Federal tornou-se obrigatória e o SINAN, atualmente, é a principal fonte de dados para a vigilância da $\mathrm{TB} 4$.

Por ser uma doença de tratamento longo, com duração mínima de seis meses, os pacientes de TB podem deslocar-se durante o período de tratamento para realizar o acompanhamento em inúmeros serviços de saúde. Para minimizar problemas em relação às informações de acompanhamento desses casos, o SINAN dispõe de rotinas específicas: remoção de duplicidades e vinculação de registros. Essas ferramentas permitem o manejo de notificações de pacientes de TB inseridas mais de uma vez durante o mesmo tratamento e a vinculação de registros de casos que foram transferidos de serviço durante o período 5 .

A qualidade das informações constitui uma importante limitação para a análise dos dados dos sistemas de informação em saúde, podendo gerar avaliações equivocadas e, com isto, comprometer o processo decisório ${ }^{6}$. Ausência de duplicidade, cobertura, completitude e confiabilidade são características dos dados ligadas à acurácia, dimensão de qualidade fundamental a ser considerada no cálculo de indicadores e identificação do perfil dos casos para a vigilância da TB 7. A garantia de qualidade para o desfecho de tratamento dos casos de TB é um desafio para a vigilância dessa doença devido à complexidade do tratamento e à possibilidade de deslocamento dos pacientes entre unidades de saúde. Além disso, os dados referentes aos óbitos de TB considerados válidos, mesmo sendo coletados pelo SINAN no desfecho de tratamento, são obtidos de outro sistema de informação, o Sistema de Informações sobre Mortalidade (SIM) 5 .

O relacionamento probabilístico ou linkage de base de dados tem sido utilizado para qualificar as informações e obter respostas mais fidedignas em relação à situação epidemiológica da TB, como pode ser visto em diversos estudos publicados nos últimos anos 8,9.

O objetivo deste trabalho é verificar a melhoria da qualidade das informações da TB após a vinculação de registros e a correção do encerramento por meio do linkage probabilístico do SINAN com o SIM, com vistas à obtenção de indicadores para o acompanhamento da TB mais confiáveis.

\section{Materiais e métodos}

Foi utilizado o SINAN como fonte de dados para os casos de TB e o SIM para os óbitos que mencionaram TB como causa básica ou associada (códigos compreendidos no intervalo de A15 a A19 da 10a revisão da Classificação Internacional de Doenças - CID-10). Os dados do SINAN e do SIM foram disponibilizados pela Secretaria de Vigilância em Saúde do Ministério da Saúde.

O estudo foi realizado em duas etapas: vinculação de registros do SINAN, para tratamento das duplicidades e dos registros com encerramento, transferência e indefinido; e linkage entre o SINAN e o SIM, para correção do encerramento nos casos de óbito. Como as bases de dados utilizadas não continham variável identificadora única entre elas, utilizou-se o método de linkage probabilístico de registros, por meio do software RecLink III (Camargo Jr. KR, Coeli CM. RecLink III versão 3.1.6.3160. Guia do usuário 2007. http:// www.iesc.ufrj.br/reclink) para as duas etapas. Esse método baseia-se na utilização conjunta de campos comuns de identificação presentes em ambos os bancos de dados, com o objetivo de identificar o quanto é provável que um par de registros faz referência a um mesmo indivíduo 10 .

Para cada processo de linkage foi executado um conjunto de etapas: padronização dos campos comuns das bases de dados a serem utilizados no linkage, criação de blocos lógicos de registros para otimização do processo, aplicação de algoritmos para a comparação aproximada de cadeias de caracteres que levam em consideração possíveis erros fonéticos e de digitação, cálculo de escores que sumarizam o grau de concordância global entre registros do mesmo par, pareamento para a definição de limiares para a classificação dos pares de registros relacionados em pares verdadeiros, não pares e pares duvidosos, e revisão manual dos pares duvidosos. 
Para o pareamento de registros, em ambos os processos de linkage, utilizaram-se as variáveis: nome do paciente e data de nascimento. A revisão manual dos pares duvidosos foi realizada por um único investigador e, durante este processo, a reclassificação destes em pares verdadeiros e não pares obedeceu ao critério de desempate: nome do paciente, data de nascimento, nome da mãe e endereço de residência. A revisão foi realizada ao final de cada passo de blocagem e os registros que permaneceram classificados como duvidosos foram considerados não pares.

\section{Vinculação de registros do SINAN}

Na esfera nacional, não é possível realizar a vinculação por meio das rotinas do SINAN devido ao elevado número de registros nas bases de dados. Assim, foi utilizado o linkage probabilístico entre os registros do SINAN com o objetivo de excluir as notificações repetidas de um mesmo paciente, que não foram retiradas ou vinculadas nas esferas municipais e estaduais.

Utilizaram-se os registros de TB do SINAN com data de diagnóstico dos anos de 2008 e 2009. As bases de dados usadas para o linkage foram construídas de acordo com a situação de encerramento dos casos. Uma base de dados (SINAN-TB1) foi formada pelos casos com situação de encerramento preenchida como transferência ou sem preenchimento, que representaram 11,8\% dos registros do período. A outra base de dados (SINAN-TB2) foi composta pelos registros com situação de encerramento preenchida como cura, abandono, óbito por TB, óbito por outras causas e tuberculose multirresistente (TB-MR). Os registros com situação de encerramento mudança de diagnóstico foram excluídos das bases de dados. Após o linkage probabilístico entre as bases SINAN-TB1 e SINAN-TB2 foram definidos como pares verdadeiros aqueles que, dentre todos os pares encontrados, apresentaram data de encerramento no SINAN-TB2 maior ou até trinta dias antes data de encerramento no SINAN-TB1, para minimizar o efeito de problemas de preenchimento da variável data de encerramento.

Foram realizados quatro passos de blocagem com as seguintes variáveis: (1) soundex do primeiro e último nomes, Unidade Federada (UF) de residência e sexo; (2) soundex do primeiro nome, UF de residência e sexo; (3) soundex do último nome, UF de residência e sexo; e (4) soundex do primeiro e último nomes.

Os escores superiores a 21,7 foram considerados pares verdadeiros e os inferiores a 0,0 não pares, permanecendo os escores intermediários como duvidosos.

\section{Linkage entre SINAN e SIM}

Com a base de dados formada a partir do primeiro linkage de dados, realizou-se o segundo processo de linkage probabilístico, entre o SINAN e o SIM, com o objetivo de identificar falhas na situação de encerramento. Foram relacionadas 170.432 notificações do SINAN dos anos de 2008 e 2009 com 22.360 Declarações de Óbito (DO) do SIM que mencionaram TB como causa básica ou associada entre 2008 e 2010 no Brasil. Optouse por utilizar um período maior para as DO do SIM com o objetivo de possibilitar a captação dos casos que foram notificados no SINAN no final de 2009 e registrados no SIM somente no ano de 2010. Foram considerados pares verdadeiros aqueles cujos registros possuíam a notificação do SINAN com data de diagnóstico mais atual, admitindo que o óbito provavelmente estava relacionado com o último evento notificado no SINAN. Para considerar que o óbito estava associado ao caso de TB, o intervalo entre a data do diagnóstico e a data do óbito deveria ser de até 270 dias entre os pares verdadeiros. Esse período foi estabelecido de acordo com o tempo definido para o encerramento de um caso novo de TB, 180 dias de tratamento mais noventa dias para o preenchimento do sistema de informação. Pares encontrados após esse período não foram considerados o mesmo evento da doença. Os pares em que a data do óbito foi antes da data do diagnóstico foram considerados pares verdadeiros.

No linkage do SINAN com o SIM foram realizados sete passos de blocagem, utilizando-se as mesmas variáveis do primeiro linkage, com o acréscimo da variável município de residência: (1) soundex do primeiro e último nomes, UF de residência e sexo; (2) soundex do primeiro nome, UF de residência e sexo; (3) soundex do último nome, UF de residência e sexo; (4) soundex do primeiro nome e sexo; (5) UF de residência e sexo; (6) município de residência e sexo e; (7) soundex do primeiro e último nomes.

Os escores superiores a 21,3 foram considerados pares verdadeiros e os inferiores a 0,2 não pares, permanecendo os escores intermediários como duvidosos (Figura 1).

\section{Análise dos resultados}

Após as duas etapas de linkage foi obtida a base de dados final qualificada com os casos de TB diagnosticados em 2008 e 2009, excluindo os registros que não tinham sido vinculados previamente e corrigindo a situação de encerramento valendo-se das informações do SIM.

Foram calculadas as frequências de cada encerramento para os casos novos de TB na base de 
Fluxograma dos processos de linkage probabilístico realizados: vinculação de registros e linkage entre Sistema de Informações de Agravos de Notificação (SINAN) e Sistema de Informações de Mortalidade (SIM).

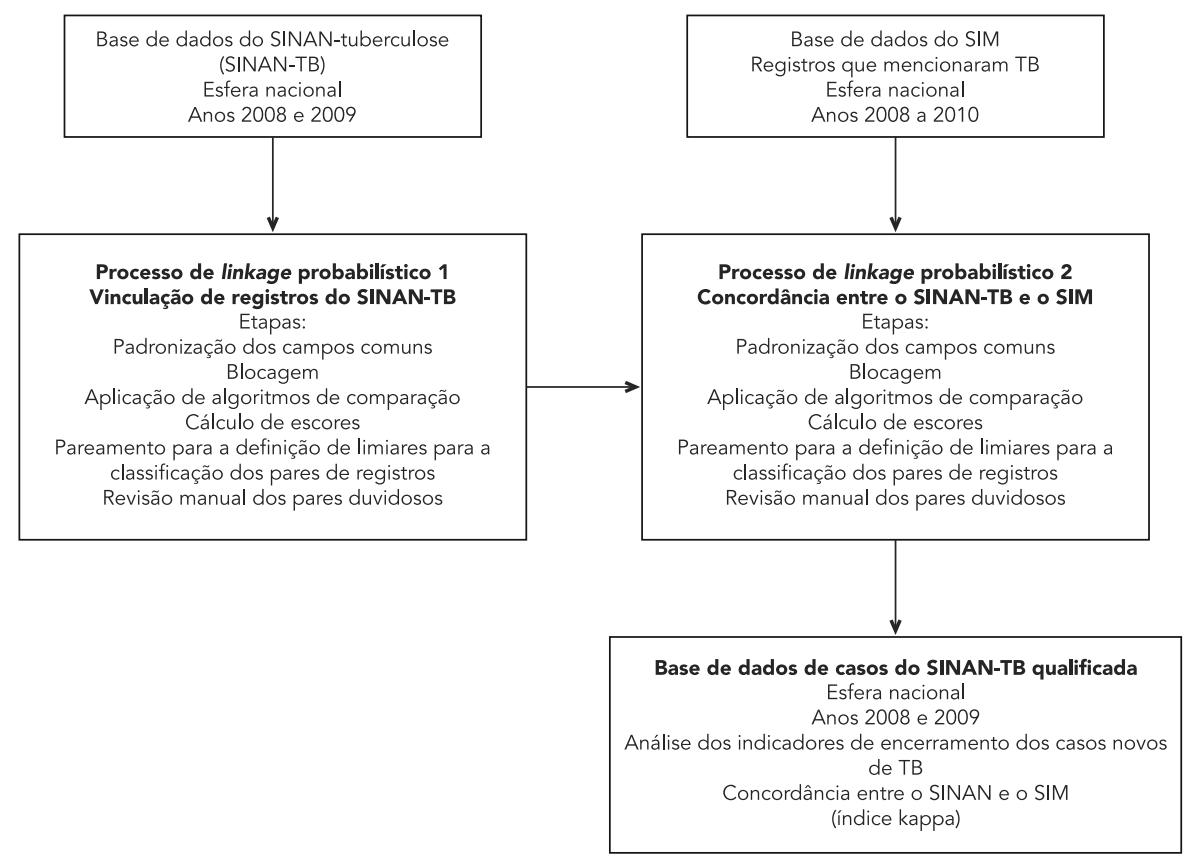

dados original e naquelas obtidas após os processos de vinculação de registros e linkage entre SINAN e SIM, para comparação. A confiabilidade do campo encerramento foi analisada por meio do índice kappa, obedecendo os parâmetros definidos por Landis \& Koch 11: pobre $(\leq 0)$; superficial $(0-0,2)$; razoável $(0,21-0,4)$; moderada $(0,41-0,6)$; substancial $(0,61-0,8)$; quase perfeita (0,81-1) e comparando as seguintes categorias: óbito por TB, óbito por outras causas e outros encerramentos/não encontrado no SIM.

Utilizou-se os softwares Microsoft Office Excel (Micrososft Corp., Estados Unidos), TabWin versão 3.2 (Departamento de Informática do SUS. http://portal.saude.gov.br/portal/se/datasus/area.cfm?id_area=732), Epi Info versão 3.5.1 (Centers for Disease Control and Prevention, Atlanta, Estados Unidos) e WinPepi 4.54 (http:// www.brixtonhealth.com/pepi4windows.html).

As bases de dados continham dados de identificação do paciente (nome e endereço), necessários para esse tipo de linkage realizado por meio de metodologia proposta pelo RecLink 9 . Informações identificadoras foram utilizadas apenas para o linkage, sendo retiradas posterior- mente para análise. O trabalho foi aprovado pelo Comitê de Ética em Pesquisa da Faculdade de Medicina da Universidade de Brasília, em 3 de julho de 2012, com o registro no 015/2012.

\section{Resultados}

Situação de encerramento dos casos novos de TB após a vinculação de registros e o linkage entre SINAN e SIM

\section{- Vinculação de registros}

A vinculação de registros valendo-se do linkage entre as bases de dados SINAN-TB1 e SINANTB2 diminuiu o percentual de casos novos com desfecho de tratamento como transferência em todas as UF nos dois anos avaliados. Para o Brasil, a redução do percentual de transferência foi de $34,8 \%$ no ano de 2008 e $35,5 \%$ no de 2009 . As maiores reduções nos anos avaliados foram para UF da Região Norte e para o Estado de Goiás, com percentuais maiores que $50 \%$. O Acre apresentou baixos percentuais de transferência 
na base original (menos de $2 \%$ ). Os estados do Rio Grande do Norte e Amapá registraram as menores variações (Tabelas 1 e 2). O percentual de cura entre os casos novos, principal indicador para avaliar a efetividade das ações de controle da TB realizadas, aumentou em todas as UF nos dois anos. Para o Brasil a variação foi em torno de 3\%. Piauí, Tocantins, Rondônia, Goiás e Maranhão foram as UF com os maiores aumentos no indicador de cura. O Acre, São Paulo, Rio Grande do Norte e Distrito Federal apresentaram as menores variações.

O restante dos encerramentos: abandono de tratamento, óbito por TB e óbito por outras causas, também registrou aumento em todas as UF após a remoção das duplicidades. Para o Brasil, o percentual de abandono entre os casos novos teve aumento, também, em torno de $3 \%$, assim com os óbitos por TB e óbitos por outras causas nos dois anos.

\section{- Linkage entre SINAN e SIM}

Nesta etapa, a situação de encerramento dos pares verdadeiros encontrados foi corrigida. Para o Brasil, o percentual de óbito por TB entre os casos novos diagnosticados aumentou, com variação em torno de $15 \%$, e os percentuais de cura, abandono, transferência e óbito por outras causas diminuíram nos dois anos avaliados (Tabelas 1 e 2).

Entre as UF, aquelas que apresentaram os maiores aumentos, acima de $40 \%$, no percentual óbito por TB foram: Rondônia, Mato Grosso e Distrito Federal no ano de 2008, e Acre, Rondônia, Roraima, Amapá e Minas Gerais em 2009. Algumas UF não alteraram os percentuais de encerramento após a realização do linkage entre SINAN e SIM. No ano de 2008, os estados de Tocantins, Espírito Santo e Santa Catarina não apresentaram alteração, e em 2009 o Estado de Tocantins e o Distrito Federal.

\section{Situação do encerramento óbito no SINAN}

Após o linkage entre SINAN e SIM foram encontrados 9.059 óbitos notificados nos dois sistemas, 7.939 óbitos ocorreram até 270 dias após a data do diagnóstico de TB e foram considerados registros relacionados ao mesmo evento. Em se tratando dos óbitos registrados com causa básica TB, $63,8 \%$ (3.047) estavam encerrados no SINAN como óbito por TB, 24\% (1.146) como óbito por outras causas, $3 \%$ (145) como cura, 3,2\% (154) como abandono, 3,6\% (172) como transferência, $0,2 \%$ (9) como TB multirresistente e 2,2\% (103) estavam com a situação de encerramento ignorada ou em branco. A mediana do tempo entre a data do diagnóstico e a data do óbito foi de 25 dias para o período estudado e a moda foi igual a zero (Tabela 3).

Entre os 2.144 óbitos com TB como causa associada e HIV como causa básica, 57,1\% (1.225) foram encerrados no SINAN como óbito por outras causas, $30,1 \%$ (645) como óbito por TB e $12,8 \%$ (274) com outros encerramentos. Dentre aqueles com causa básica doenças do aparelho respiratório, $36,5 \%$ (54) foram encerrados no SINAN como óbito por TB e 50,7\% (75) como óbito por outras causas. Os registros com causa básica neoplasia ou doença do aparelho circulatório apresentaram os maiores percentuais de encerramento no SINAN como óbito por outras causas, $62,3 \%$ e $60,2 \%$, respectivamente. Entre os registros notificados no SIM como óbito por outras doenças infecciosas (102), 53,9\% (55) foram encerrados no SINAN como óbito por outras causas. Outras causas básicas de óbito, como doenças do aparelho digestivo, doenças endócrinas e nutricionais e metabólicas, apresentaram o percentual de encerramento nas notificações do SINAN como óbito por outras causas, variando de $40,4 \%$ a $54,1 \%$.

O índice kappa entre SINAN e SIM para todos os registros do SINAN de 2008 e 2009 foi de 0,55, considerado moderado.

\section{Discussão}

O linkage probabilístico entre as bases de dados contribuiu com a melhoria da qualidade do SINAN, com efeitos positivos para as informações sobre a TB no Brasil. Embora as rotinas do SINAN, de remoção de duplicidade e vinculação de registros, estejam disseminadas nos Programas de Controle da TB (PCT) estaduais e municipais e devam ser consideradas uma atividade de caráter permanente dos técnicos responsáveis pela vigilância da TB 5 , os resultados apontam que elas ainda não estão sendo realizadas adequadamente. A redução dos registros duplicados com situação de encerramento indefinida ou transferência foi considerável em praticamente todas as UF do país, gerando aumento do percentual de cura da ordem de $3 \%$ para os casos novos. Esse resultado aponta para a melhoria da informação do encerramento, quando são comparados com os maiores percentuais obtidos após processos de qualificação realizados em anos anteriores por outros autores 12,13. Entretanto, os percentuais de cura para a maioria das UF ainda estão distantes do patamar de $85 \%$ sugerido pela OMS 1. Para a base de dados nacional, a situação de encerramento preenchida como transferência deveria ser transitória e não manter-se após a finalização do tratamento do 
Tabela 1

Situação de encerramento dos casos novos de tuberculose (TB) antes e após a remoção de duplicidade e o linkage entre Sistema de Informações de Agravos de Notificação (SINAN) e Sistemas de Informações sobre Mortalidade (SIM), por Unidade Federada (UF), Brasil, 2008.

\begin{tabular}{|c|c|c|c|c|c|c|c|c|c|c|c|c|c|c|c|}
\hline \multirow{4}{*}{$\begin{array}{l}\text { UF/ } \\
\text { Região }\end{array}$} & \multicolumn{3}{|c|}{ Cura (\%) } & \multicolumn{3}{|c|}{ Abandono (\%) } & \multicolumn{3}{|c|}{ Transferência (\%) } & \multicolumn{3}{|c|}{ Óbito por TB (\%) } & \multirow{2}{*}{\multicolumn{3}{|c|}{$\begin{array}{c}\text { Óbito por outras causas } \\
(\%)\end{array}$}} \\
\hline & & & & & & & & & & & & & & & \\
\hline & Original & Após & Após & Original & Após & Após & Original & Após & Após & Original & Após & Após & Original & Após & Após \\
\hline & & L1 * & L2 ** & & $\mathrm{L} 1$ * & L2 ** & & $\mathrm{L} 1$ * & L2 ** & & $\mathrm{L} 1$ * & $\mathrm{L} 2$ ** & & $\mathrm{L} 1$ * & $\mathrm{L} 2$ ** \\
\hline Norte & 71,2 & 74,0 & 73,9 & 10,5 & 10,9 & 10,7 & 8,2 & 3,1 & 4,7 & 3,0 & 2,8 & 3,7 & 2,7 & 4,9 & 2,8 \\
\hline$A C$ & 86,5 & 87,5 & 87,5 & 7,7 & 7,7 & 7,7 & 1,1 & 0,0 & 0,0 & 3,3 & 3,3 & 2,6 & 1,1 & 1,1 & 1,8 \\
\hline AP & 65,2 & 67,6 & 66,8 & 11,6 & 12,0 & 11,9 & 17,2 & 14,2 & 14,2 & 1,3 & 1,3 & 1,8 & 3,9 & 4,0 & 4,4 \\
\hline AM & 68,4 & 71,9 & 71,8 & 9,5 & 10,0 & 9,8 & 7,9 & 3,8 & 3,7 & 4,3 & 4,6 & 5,4 & 2,6 & 2,8 & 2,5 \\
\hline PA & 71,3 & 73,8 & 73,6 & 11,9 & 12,3 & 12,0 & 8,3 & 5,4 & 5,3 & 2,5 & 2,6 & 2,8 & 2,5 & 2,5 & 2,8 \\
\hline $\mathrm{RO}$ & 74,4 & 78,0 & 78,0 & 10,6 & 11,1 & 10,9 & 8,9 & 4,6 & 4,1 & 1,5 & 1,5 & 3,3 & 2,3 & 2,4 & 1,3 \\
\hline RR & 80,1 & 83,2 & 83,2 & 4,4 & 4,6 & 4,6 & 5,1 & 1,5 & 1,5 & 2,2 & 2,3 & 3,1 & 6,6 & 6,9 & 6,1 \\
\hline TO & 75,0 & 76,8 & 76,8 & 4,7 & 4,8 & 4,8 & 10,5 & 8,3 & 8,3 & 1,2 & 1,2 & 1,2 & 7,0 & 7,1 & 7,1 \\
\hline Nordeste & 71,5 & 74,2 & 74,0 & 9,0 & 9,3 & 9,2 & 9,8 & 6,7 & 6,5 & 3,2 & 3,3 & 3,9 & 2,9 & 3,0 & 3,0 \\
\hline $\mathrm{AL}$ & 74,1 & 76,0 & 75,9 & 10,1 & 10,4 & 10,2 & 6,8 & 4,7 & 4,6 & 3,6 & 3,7 & 3,4 & 2,5 & 2,6 & 3,2 \\
\hline BA & 72,9 & 75,0 & 74,8 & 6,7 & 7,0 & 6,9 & 8,3 & 6,2 & 6,1 & 2,6 & 2,6 & 3,4 & 3,0 & 3,0 & 2,9 \\
\hline CE & 76,7 & 79,4 & 79,1 & 8,5 & 8,8 & 8,7 & 7,3 & 4,4 & 4,2 & 2,8 & 2,9 & 3,5 & 2,3 & 2,4 & 2,5 \\
\hline MA & 73,9 & 78,2 & 77,7 & 8,7 & 9,2 & 9,0 & 10,8 & 5,6 & 5,5 & 2,4 & 2,5 & 3,5 & 2,8 & 3,0 & 2,8 \\
\hline PB & 64,4 & 67,2 & 67,1 & 13,0 & 13,8 & 13,4 & 15,1 & 11,2 & 11,1 & 2,9 & 3,0 & 3,6 & 2,3 & 2,4 & 2,5 \\
\hline PE & 65,3 & 68,1 & 67,9 & 11,4 & 11,8 & 11,6 & 11,6 & 8,2 & 7,6 & 4,2 & 4,4 & 4,9 & 3,6 & 3,8 & 4,2 \\
\hline $\mathrm{Pl}$ & 66,3 & 71,4 & 71,2 & 4,1 & 4,4 & 4,3 & 20,0 & 13,8 & 13,7 & 3,5 & 3,9 & 5,1 & 3,0 & 3,2 & 2,5 \\
\hline $\mathrm{RN}$ & 71,4 & 72,3 & 72,1 & 9,1 & 9,2 & 9,2 & 10,6 & 9,4 & 8,6 & 4,1 & 4,2 & 5,3 & 2,5 & 2,6 & 2,6 \\
\hline SE & 75,0 & 76,2 & 76,0 & 13,8 & 14,0 & 13,8 & 4,1 & 2,8 & 2,8 & 4,6 & 4,7 & 4,3 & 2,2 & 2,2 & 2,9 \\
\hline Sudeste & 73,2 & 74,5 & 74,4 & 10,3 & 10,6 & 10,5 & 3,7 & 2,5 & 2,4 & 3,3 & 3,3 & 3,9 & 4,4 & 4,4 & 4,3 \\
\hline ES & 80,8 & 82,3 & 82,3 & 5,6 & 5,8 & 5,8 & 6,0 & 4,3 & 4,2 & 3,6 & 3,6 & 3,6 & 2,5 & 2,5 & 2,7 \\
\hline MG & 74,9 & 76,6 & 76,3 & 8,8 & 9,0 & 8,9 & 5,8 & 3,8 & 3,6 & 3,5 & 3,6 & 4,4 & 6,5 & 6,6 & 6,4 \\
\hline RJ & 65,4 & 67,8 & 67,7 & 11,6 & 12,1 & 11,9 & 6,3 & 4,2 & 4,1 & 3,7 & 3,8 & 3,7 & 1,6 & 1,7 & 2,6 \\
\hline SP & 77,7 & 78,0 & 77,9 & 10,3 & 10,3 & 10,3 & 1,1 & 0,8 & 0,8 & 2,9 & 2,9 & 3,9 & 6,0 & 6,0 & 5,2 \\
\hline Sul & 70,7 & 73,5 & 73,5 & 9,4 & 9,8 & 9,7 & 8,8 & 5,3 & 5,1 & 3,7 & 3,8 & 4,0 & 5,2 & 5,4 & 5,6 \\
\hline PR & 73,9 & 75,6 & 75,5 & 8,4 & 8,6 & 8,5 & 7,6 & 5,6 & 5,4 & 3,6 & 3,7 & 4,1 & 5,6 & 5,7 & 5,6 \\
\hline RS & 68,2 & 71,6 & 71,5 & 10,4 & 11,0 & 10,7 & 9,4 & 5,0 & 4,8 & 3,8 & 3,9 & 4,2 & 5,1 & 5,4 & 5,7 \\
\hline SC & 73,2 & 75,6 & 75,6 & 8,2 & 8,6 & 8,5 & 8,7 & 5,7 & 5,4 & 3,4 & 3,5 & 3,5 & 4,8 & 4,9 & 5,3 \\
\hline $\begin{array}{l}\text { Centro- } \\
\text { oeste }\end{array}$ & 75,7 & 78,4 & 78,1 & 7,0 & 7,3 & 7,2 & 8,3 & 5,1 & 5,0 & 3,0 & 3,1 & 4,3 & 4,4 & 4,6 & 4,1 \\
\hline DF & 82,2 & 85,1 & 84,8 & 3,7 & 3,8 & 3,8 & 9,9 & 6,7 & 6,4 & 0,6 & 0,6 & 0,9 & 3,4 & 3,5 & 3,8 \\
\hline $\mathrm{GO}$ & 73,1 & 77,4 & 77,4 & 7,9 & 8,4 & 8,2 & 9,4 & 4,4 & 4,3 & 3,0 & 3,1 & 4,4 & 5,1 & 5,4 & 4,6 \\
\hline MS & 76,9 & 79,6 & 79,0 & 7,6 & 7,9 & 7,7 & 9,6 & 6,4 & 6,2 & 2,2 & 2,3 & 3,9 & 3,4 & 3,5 & 2,8 \\
\hline MT & 74,1 & 75,4 & 74,9 & 6,9 & 7,0 & 7,0 & 5,1 & 3,7 & 3,5 & 5,1 & 5,2 & 6,2 & 5,5 & 5,6 & 5,1 \\
\hline Brasil & 72,3 & 74,4 & 74,2 & 9,7 & 10,0 & 9,9 & 6,7 & 4,4 & 4,2 & 3,3 & 3,4 & 3,9 & 3,9 & 4,0 & 4,0 \\
\hline
\end{tabular}

AC: Acre; AL: Alagoas; AM: Amazonas; AP: Amapá; BA: Bahia; CE: Ceará; DF: Distrito Federal; ES: Espírito Santo; GO: Goiás; MA: Maranhão;

MG: Minas Gerais; MS: Mato Grosso do Sul; MT: Mato Grosso; PA: Pará; PB: Paraíba; PR: Paraná; PE: Pernambuco; PI: Piauí; RO: Rondônia;

RJ: Rio de Janeiro; RN: Rio Grande do Norte; RR: Roraima; RS: Rio Grande do Sul; SC: Santa Catarina; SE: Sergipe; SP: São Paulo; TO: Tocantins.

* L1: processo de linkage para vinculação de registros;

** L2: processo de linkage entre SINAN e SIM.

paciente. No entanto, essa não é uma realidade, uma vez que verificou-se a redução expressiva do percentual de transferência após o primeiro linkage, ou ainda manteve-se elevado após este processo.
Os estados de São Paulo, Acre, Roraima e Mato Grosso do Sul apresentaram qualidade nos dados disponíveis, sugerindo adequada realização das rotinas de análise de duplicidade e vinculação de registros. Apesar de São Paulo ser a UF com 
Situação de encerramento dos casos novos de tuberculose antes e após a remoção de duplicidade e o linkage entre Sistema de Informações de Agravos de Notificação (SINAN) e Sistemas de Informações sobre Mortalidade (SIM), por Unidade Federada (UF), Brasil, 2009.

\begin{tabular}{|c|c|c|c|c|c|c|c|c|c|c|c|c|c|c|c|}
\hline \multirow{3}{*}{$\begin{array}{l}\text { UF/ } \\
\text { Região }\end{array}$} & \multicolumn{3}{|c|}{ Cura (\%) } & \multicolumn{3}{|c|}{ Abandono (\%) } & \multicolumn{3}{|c|}{ Transferência (\%) } & \multicolumn{3}{|c|}{ Óbito por TB (\%) } & \multicolumn{3}{|c|}{ Óbito por outras causas (\%) } \\
\hline & Original & Após & Após & Original & Após & Após & Original & Após & Após & Original & Após & Após & Original & Após & Após \\
\hline & & L1 * & L2 ** & & $\mathrm{L} 1$ * & L2 ** & & $\mathrm{L} 1$ * & L2 ** & & L1 * & L2 ** & & L1 * & L2 ** \\
\hline Norte & 73,4 & 76,4 & 76,3 & 9,6 & 10,0 & 9,8 & 8,9 & 5,3 & 5,2 & 3,0 & 3,1 & 3,5 & 2,7 & 2,9 & 3,0 \\
\hline$A C$ & 90,7 & 90,9 & 90,9 & 4,4 & 4,4 & 4,4 & 1,2 & 0,9 & 0,9 & 0,6 & 0,6 & 1,3 & 1,9 & 1,9 & 1,3 \\
\hline$A P$ & 67,1 & 70,7 & 70,7 & 11,0 & 11,5 & 11,1 & 20,1 & 15,9 & 15,9 & 0,9 & 1,0 & 1,4 & 0,9 & 1,0 & 1,0 \\
\hline AM & 75,0 & 77,6 & 77,6 & 10,0 & 10,4 & 10,2 & 6,6 & 3,5 & 3,4 & 5,4 & 5,6 & 5,7 & 2,1 & 2,1 & 2,3 \\
\hline PA & 71,7 & 74,8 & 74,7 & 9,8 & 10,3 & 10,0 & 9,1 & 5,5 & 5,3 & 2,1 & 2,1 & 2,7 & 3,2 & 3,3 & 3,5 \\
\hline $\mathrm{RO}$ & 68,7 & 72,9 & 73,0 & 10,7 & 11,3 & 11,1 & 16,3 & 11,3 & 11,1 & 0,9 & 0,9 & 1,8 & 3,0 & 3,2 & 2,5 \\
\hline $\mathrm{RR}$ & 82,6 & 84,5 & 84,5 & 8,3 & 8,5 & 8,5 & 3,8 & 1,6 & 1,6 & 2,3 & 2,3 & 0,8 & 3,0 & 3,1 & 4,7 \\
\hline TO & 72,6 & 78,1 & 77,5 & 4,0 & 4,3 & 4,3 & 11,9 & 5,3 & 5,3 & 5,5 & 5,9 & 5,9 & 5,5 & 5,9 & 6,4 \\
\hline Nordeste & 68,6 & 71,7 & 71,6 & 8,8 & 9,2 & 9,1 & 11,4 & 7,7 & 7,4 & 3,4 & 3,5 & 3,9 & 3,3 & 3,5 & 3,7 \\
\hline $\mathrm{AL}$ & 69,0 & 71,9 & 71,5 & 10,0 & 10,6 & 10,5 & 9,0 & 5,6 & 5,2 & 4,8 & 5,0 & 4,4 & 3,0 & 3,1 & 4,7 \\
\hline BA & 69,7 & 73,3 & 73,1 & 6,8 & 7,2 & 7,1 & 11,5 & 7,5 & 7,2 & 3,0 & 3,1 & 3,8 & 3,3 & 3,5 & 3,5 \\
\hline CE & 73,6 & 76,3 & 76,3 & 8,8 & 9,2 & 9,0 & 9,3 & 6,1 & 5,9 & 3,3 & 3,4 & 3,5 & 2,1 & 2,1 & 2,6 \\
\hline MA & 71,9 & 75,7 & 75,4 & 11,6 & 12,2 & 11,9 & 9,9 & 5,3 & 5,2 & 2,8 & 3,0 & 4,0 & 3,0 & 3,1 & 2,8 \\
\hline PB & 63,9 & 67,2 & 67,1 & 8,2 & 8,6 & 8,6 & 17,6 & 13,4 & 13,3 & 2,9 & 3,1 & 2,8 & 2,9 & 3,1 & 3,6 \\
\hline PE & 62,0 & 64,4 & 64,3 & 10,8 & 11,4 & 11,2 & 13,2 & 9,8 & 9,4 & 3,6 & 3,8 & 4,3 & 5,0 & 5,2 & 5,5 \\
\hline $\mathrm{Pl}$ & 62,3 & 67,6 & 67,1 & 3,1 & 3,3 & 3,3 & 16,0 & 9,6 & 9,1 & 2,9 & 3,2 & 4,1 & 3,5 & 3,8 & 4,0 \\
\hline $\mathrm{RN}$ & 70,3 & 71,8 & 71,6 & 9,6 & 9,8 & 9,8 & 10,6 & 8,9 & 8,5 & 3,5 & 3,6 & 3,5 & 2,6 & 2,6 & 3,4 \\
\hline SE & 74,8 & 76,7 & 76,7 & 9,5 & 9,7 & 9,7 & 5,6 & 3,8 & 3,8 & 5,4 & 5,6 & 5,7 & 4,2 & 4,3 & 4,1 \\
\hline Sudeste & 73,5 & 74,7 & 74,6 & 11,5 & 11,7 & 11,6 & 3,7 & 2,4 & 2,4 & 3,1 & 3,1 & 3,7 & 4,7 & 4,7 & 4,6 \\
\hline ES & 78,9 & 80,5 & 80,5 & 7,4 & 7,5 & 7,5 & 5,7 & 3,8 & 3,9 & 3,5 & 3,6 & 3,8 & 3,8 & 3,8 & 3,7 \\
\hline MG & 73,8 & 75,2 & 74,9 & 10,0 & 10,3 & 10,2 & 5,6 & 3,8 & 3,6 & 3,3 & 3,4 & 4,7 & 6,7 & 6,9 & 6,0 \\
\hline RJ & 67,4 & 69,4 & 69,4 & 14,1 & 14,6 & 14,3 & 6,1 & 4,0 & 4,0 & 3,3 & 3,4 & 3,3 & 2,8 & 2,9 & 3,6 \\
\hline SP & 77,5 & 78,0 & 77,9 & 10,3 & 10,3 & 10,3 & 1,3 & 0,8 & 0,8 & 2,8 & 2,8 & 3,6 & 5,5 & 5,6 & 5,0 \\
\hline Sul & 70,1 & 73,0 & 72,8 & 9,2 & 9,6 & 9,3 & 9,0 & 5,4 & 5,3 & 3,0 & 3,1 & 3,4 & 5,8 & 6,0 & 6,4 \\
\hline PR & 73,2 & 75,6 & 75,2 & 7,4 & 7,7 & 7,6 & 7,4 & 4,4 & 4,5 & 3,4 & 3,5 & 3,7 & 5,7 & 5,9 & 6,2 \\
\hline RS & 66,8 & 70,4 & 70,2 & 10,7 & 11,3 & 10,8 & 10,5 & 6,0 & 5,8 & 2,8 & 2,9 & 3,5 & 5,8 & 6,1 & 6,4 \\
\hline SC & 75,9 & 77,4 & 77,4 & 7,1 & 7,4 & 7,3 & 7,0 & 5,2 & 5,0 & 2,9 & 3,0 & 2,7 & 5,8 & 5,9 & 6,4 \\
\hline $\begin{array}{l}\text { Centro- } \\
\text { oeste }\end{array}$ & 72,5 & 74,6 & 74,3 & 7,7 & 7,9 & 7,9 & 7,3 & 4,8 & 4,7 & 4,1 & 4,2 & 4,7 & 5,2 & 5,3 & 5,3 \\
\hline DF & 86,3 & 87,5 & 87,2 & 2,5 & 2,5 & 2,5 & 5,3 & 3,9 & 3,9 & 0,7 & 0,7 & 0,7 & 4,2 & 4,3 & 4,6 \\
\hline GO & 70,7 & 72,9 & 72,9 & 8,7 & 9,0 & 8,8 & 7,7 & 5,2 & 5,1 & 4,1 & 4,2 & 4,9 & 5,3 & 5,5 & 5,1 \\
\hline MS & 69,3 & 70,9 & 75,4 & 8,4 & 8,6 & 7,9 & 4,9 & 2,8 & 6,3 & 6,5 & 6,7 & 3,9 & 6,2 & 6,4 & 4,5 \\
\hline MT & 72,9 & 75,8 & 70,5 & 7,7 & 8,0 & 8,6 & 9,8 & 6,5 & 2,8 & 2,9 & 3,1 & 6,8 & 4,4 & 4,5 & 6,7 \\
\hline Brasil & 71,6 & 73,8 & 73,7 & 10,1 & 10,4 & 10,3 & 7,2 & 4,7 & 4,5 & 3,2 & 3,3 & 3,7 & 4,3 & 4,4 & 4,4 \\
\hline
\end{tabular}

AC: Acre; AL: Alagoas; AM: Amazonas; AP: Amapá; BA: Bahia; CE: Ceará; DF: Distrito Federal; ES: Espírito Santo; GO: Goiás; MA: Maranhão; MG: Minas Gerais; MS: Mato Grosso do Sul; MT: Mato Grosso; PA: Pará; PB: Paraíba; PR: Paraná; PE: Pernambuco; Pl: Piauí; RO: Rondônia; RJ: Rio de Janeiro; RN: Rio Grande do Norte; RR: Roraima; RS: Rio Grande do Sul; SC: Santa Catarina; SE: Sergipe; SP: São Paulo; TO: Tocantins. * L1: processo de linkage para vinculação de registros;

** L2: processo de linkage entre SINAN e SIM.

o maior número de casos, a base de dados original deste estado apresentou baixo percentual de casos novos encerrados como transferência 2 . Deve-se considerar que o sistema de informação dessa UF é diferenciado do resto do país. O es- tado utiliza o Sistema de Controle de Pacientes com TB (TBWEB), sistema online, que possui um registro único por paciente, fato que minimiza o registro de transferências (Divisão de Tuberculose, Centro de Vigilância Epidemiológica 
Tabela 3

Resultados da situação de notificação dos óbitos por/com tuberculose obtidos após o linkage entre Sistema de Informações de Agravos de Notificação (SINAN) e Sistemas de Informações sobre Mortalidade (SIM), Brasil, 2008 a 2010.

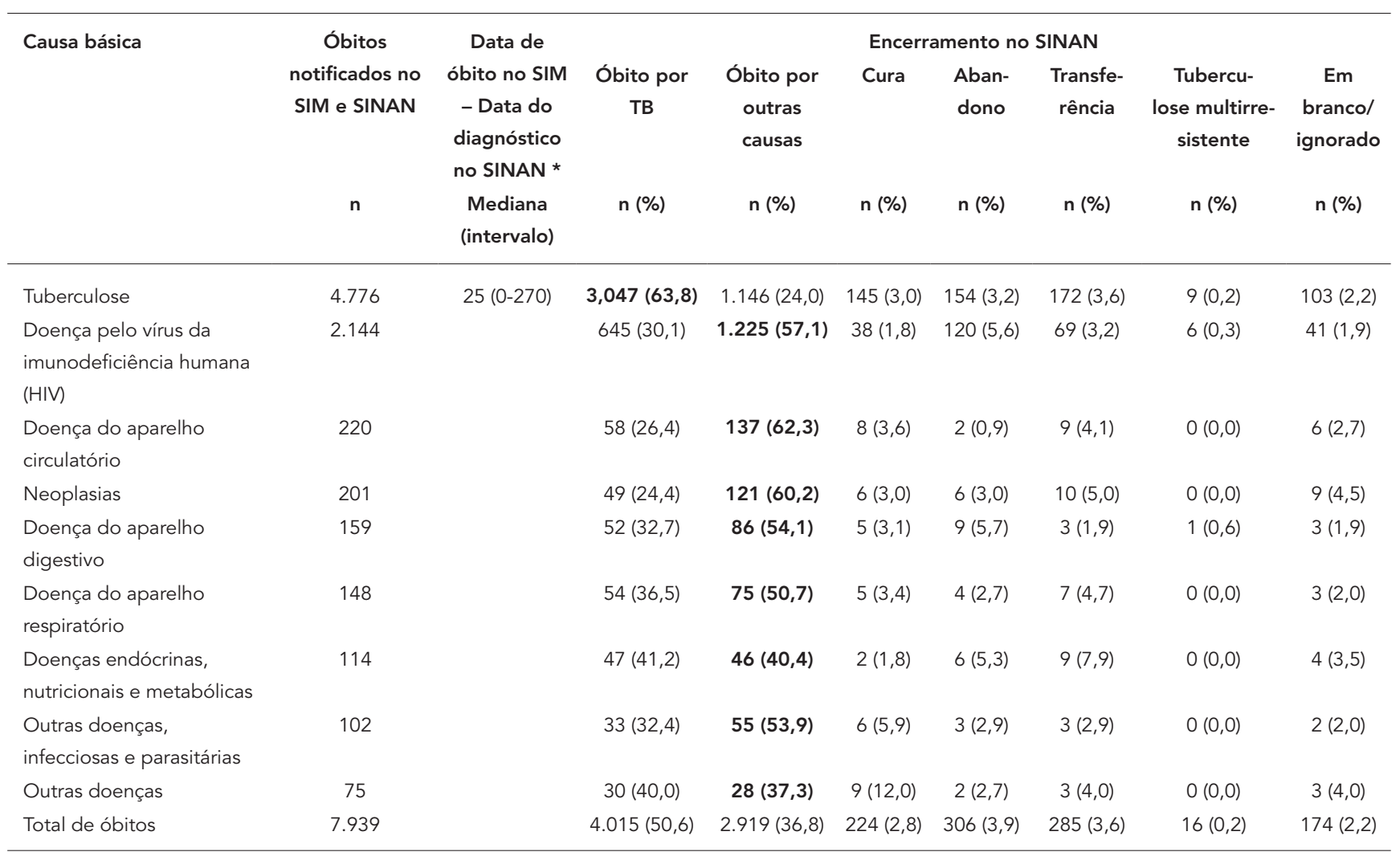

* Data em dias. Para o cálculo da mediana foram excluídos os casos com data do óbito menor que a data do diagnóstico por considerar erros de digitação.

Professor Alexandre Vranjac, Coordenadoria de Controle de Doenças, Secretaria Estadual de Saúde de São Paulo. TBWEB versão 1.6. http://www. cve.saude.sp.gov.br/htm/TB/mat_tec/manu ais/DvTBC_TBWEB_2008.pdf, acessado em 24/ Fev/2013) e dispensa a necessidade de realizar as rotinas de melhoria da qualidade do SINAN. Entretanto, esse não é o caso das outras citadas, sugerindo que a realização sistemática das rotinas de duplicidades e vinculação de registros pelos PCT contribui para a melhoria da qualidade da base de dados.

Embora o SIM seja considerado referência para a vigilância dos óbitos por TB 14 e a OMS recomende a sua utilização para a recuperação dos casos não notificados no SINAN 15 , estudos têm apontado a ocorrência de subnotificação de óbitos de TB no sistema de vigilância do país 9,13,14,16,17. Trabalhos de abrangência nacional e local descreveram que apenas $70 \%$ dos casos registrados no SIM foram encerrados no SINAN como óbito 13,18. Mesmo com períodos e recortes de análise diferentes, os resultados foram seme- lhantes aos encontrados neste estudo (63,8\%), sinalizando a necessidade de uma intervenção específica para a mudança desse quadro.

A análise de concordância entre o desfecho dos casos de TB no SINAN e o registro do óbito no SIM foi moderada 11. A falta de concordância encontrada entre SIM e SINAN demonstra a necessidade de melhorar a integração e o fluxo de informação entre os sistemas. Exceção feita para Tocantins, Santa Catarina e Espírito Santo, que parecem ter rotina para o acompanhamento das informações de TB nos dois sistemas. Essa baixa concordância pode, ainda, ser explicada pela ausência de critérios para a classificação do encerramento óbito no SINAN, diferente do SIM, que possui um algoritmo de classificação das causas reconhecido internacionalmente 19 .

Para relacionar o óbito registrado no SIM à notificação no SINAN considerou-se um intervalo de 270 dias, diferentemente de outro estudo que considerou o prazo de um ano para relacionar as duas notificações 14. Embora possa ter ocorrido subestimação de pares verdadeiros com 
esse período reduzido, a chance de certeza de um óbito estar relacionados à notificação no SINAN aumenta, além desse ser o intervalo considerado máximo para encerrar um caso de TB notificado no SINAN.

A mediana de trinta dias entre a data do diagnóstico no SINAN e a data de óbito no SIM remete à dificuldade de acesso aos serviços de saúde para o diagnóstico e tratamento. A alta proporção de óbitos ocorridos pouco depois do início do tratamento foi verificada por outros autores 20,21, e revela as limitações dos serviços de saúde em identificar precocemente e tratar oportunamente uma parcela significativa dos portadores de TB 22,23, reforçada neste estudo pelo valor da moda igual a zero.

Algumas limitações devem ser citadas. Foi utilizado o linkage probabilístico para a identificação de pares e o preenchimento das variáveis de identificação, essencial nesta metodologia que, muitas vezes, apresentam problemas de preenchimento 17 . Sabe-se, também, que há falhas no preenchimento das datas, principalmente aquelas que constam no boletim de acompanhamento 24 . Porém, trabalhos que avaliaram os resultados do processo de linkage com bases de mortalidade demonstraram que a realização do linkage probabilístico é satisfatória 25,26,27. Como o encerramento foi utilizado para dividir a base de dados na primeira etapa do trabalho, erros no preenchimento do encerramento podem ter impossibilitado a identificação de pares verdadeiros.

Os resultados encontrados descrevem uma situação de alerta no que se refere à qualidade dos dados de encerramento dos casos do SINAN.
Além de não se conhecer a informação verdadeira sobre a situação da TB no Brasil, a falta de qualidade atinge o indicador "percentual de casos novos curados" presente nas principais pactuações entre as esferas de governo, dificultando o alcance das metas estabelecidas 28 . Ainda são necessários investimentos nas atividades de vigilância, fluxos de informação e preenchimento dos sistemas de informação, para contribuir com o controle da doença. O linkage de bases de dados pode ser usado como uma importante ferramenta tanto em pesquisas epidemiológicas como na rotina dos serviços de vigilância em saúde para qualificar as informações, auxiliando na tomada de decisões.

Outras fontes de dados, além do SIM, podem ser utilizadas para auxiliar na melhoria da qualidade das informações de TB com o uso do linkage, como o livro de registros e acompanhamento dos casos de TB, exames laboratoriais e autorização de internação hospitalar, por exemplo 9,16,29. Para que os serviços de saúde utilizem essa ferramenta, é importante investir em qualificação dos técnicos para o manuseio de base de dados e realização de linkage. Mais do que estimular o uso de ferramentas robustas é fundamental desenvolver a capacidade de análise dos dados disponíveis nos sistemas de informação. Em municípios e estados onde o número de casos notificados é reduzido, muitas vezes apenas uma adaptação no fluxo de informação pode minimizar ou corrigir falhas existentes.

Por fim, é necessário adequar o SINAN às especificidades de uma doença de tratamento de longa duração e concretizar as melhorias que vêm sendo amplamente apontadas na literatura. 


\section{Resumen}

Este trabajo verificará la mejora de la calidad de la información sobre la tuberculosis (TB), después de vincular los registros y la corrección del cierre de los casos, a través del vínculo entre el Sistema de Información de Agravios y Notificaciones (SINAN) y el Sistema de Información sobre Mortalidad (SIM). Para vincular los registros se efectuó un linkage entre los registros del SINAN de Brasil, durante el periodo de 2008-2009, con el objetivo de eliminar las notificaciones aún existentes en las prácticas del SINAN por parte de los estados y municipios. Las bases de datos se construyeron de acuerdo con el cierre de casos. Para el linkage entre SINAN y SIM se utilizó el resultado del vínculo de registros de la base de datos y los registros del SIM que han citado la TB como causa principal o asociada, entre 2008-2010. El vínculo de registros ha reducido el porcentaje de nuevos casos con el cierre por transferencia, con una variación de alrededor un 35,0\%. Posterior al linkage entre SINAN y SIM, el porcentaje de fallecimientos por TB entre los casos ha aumentado. Los resultados describen una situación de alerta en lo que se refiere a la calidad de los datos de cierre de los casos en SINAN.

Tuberculosis; Sistemas de Información; Causas de Muerte

\section{Colaboradores}

P. Bartholomay participou da concepção e projeto do trabalho, análise e interpretação dos dados e redação do artigo. G. P. Oliveira e R. S. Pinheiro colaboraram na discussão e elaboração do artigo. A. M. N. Vasconcelos participou da concepção e projeto do trabalho e contribuiu na elaboração do artigo.

\section{Agradecimentos}

Ao Programa Nacional de Controle da Tuberculose (PNCT), pelo acesso ao banco de dados para a realização deste trabalho e à Capes, pelo apoio financeiro.

\section{Referências}

1. World Health Organization. Global tuberculosis control 2012. Geneva: World Health Organization; 2012.

2. Departamento de Vigilância das Doenças Transmissíveis, Secretaria de Vigilância em Saúde, Ministério da Saúde. Apresentação padrão: Programa Nacional de Controle da Tuberculose. http:// portal.saude.gov.br/PORTAL/SAUDE/PROFISSIO NAL/AREA.CFM?ID_AREA=1527 (acessado em 03/ Mar/2013).
3. Teixeira MG, Penna GO, Risi JB, Penna ML, Alvim MF, Moraes JC, et al. Seleção das doenças de notificação compulsória: critérios e recomendações para as três esferas de governo. Inf Epidemiol SUS 1998; 7:7-28.

4. Laguardia J, Domingues CMA, Carvalho C, Lauerman CR, Macario E, Glatt R. Sistema de Informação de Agravos de Notificação (SINAN): desafios no desenvolvimento de um sistema de informação em saúde. Epidemiol Serv Saúde 2004; 13:135-47. 
5. Departamento de Vigilância Epidemiológica, Secretaria de Vigilância em Saúde, Ministério da Saúde. Manual de recomendações para o controle da tuberculose no Brasil. Brasília: Ministério da Saúde; 2011. (Série A: Manuais e Normas Técnicas).

6. Departamento de Análise de Situação em Saúde, Secretaria de Vigilância em Saúde, Ministério da Saúde. Saúde Brasil 2010: uma análise da situação de saúde e de evidências selecionadas de impacto de ações de vigilância em saúde. Brasília: Ministério da Saúde; 2011. (Série G. Estatística e Informação em Saúde).

7. Lima CRA, Schramm JMA, Coeli CM. Gerenciamento da qualidade da informação: uma abordagem para o setor saúde. Cad Saúde Colet (Rio J.) 2010; 18:19-31.

8. Silva JPL, Travassos C, Vasconcellos MM, Campos LM. Revisão sistemática sobre encadeamento ou linkage de bases de dados secundários para uso em pesquisa em saúde no Brasil. Cad Saúde Colet (Rio J.) 2006; 14:197-224.

9. Pinheiro RS, Andrade VL, Oliveira GP. Subnotificação da tuberculose no Sistema de Informação de Agravos de Notificação (SINAN): abandono primário de bacilíferos e captação de casos em outras fontes de informação usando linkage probabilístico. Cad Saúde Pública 2012; 28:1559-68.

10. Camargo Jr. KR, Coeli CM. Reclink: aplicativo para o relacionamento de bases de dados, implementando o método probabilistic record linkage. Cad Saúde Pública 2000; 16:439-47.

11. Landis JR, Koch GG. The measurement of observer agreement for categorical data. Biometrics 1977; 33:159-74.

12. Bierrenbach AL, Oliveira GP, Codenotti S, Gomes AB, Stevens AP. Duplicates and misclassification of tuberculosis notification records in Brazil, 20012007. Int J Tuberc Lung Dis 2010; 14:593-9.

13. Sousa MGG, Andrade JRS, Dantas CF, Cardoso MD. Investigação de óbitos por tuberculose, ocorridos na Região Metropolitana do Recife (PE), registrados no Sistema de Informação de Mortalidade, entre 2001 e 2008. Cad Saúde Colet (Rio J.) 2012; 20:153-60.

14. Selig L, Kritski AL, Cascao AM, Braga JU, Trajman A, de Carvalho RM. Proposta de vigilância de óbitos por tuberculose em sistemas de informação. Rev Saúde Pública 2010; 44:1072-8.

15. World Health Organization. Global tuberculosis control 2011. Geneva: World Health Organization; 2011.

16. Sousa LM, Pinheiro RS. Óbitos e internações por tuberculose não notificados no Município do Rio de Janeiro. Rev Saúde Pública 2011; 45:31-9.

17. Oliveira GP, Pinheiro RS, Coeli CM, Barreira D, Codenotti SB. Uso do sistema de informação sobre mortalidade para identificar subnotificação de casos de tuberculose no Brasil. Rev Bras Epidemiol 2012; 15:468-77.
18. Oliveira GP, Pinheiro RS, Coeli CM, Codenotti SB, Barreira D. Linkage entre SIM e SINAN para a melhoria da qualidade dos dados do sistema de informação da tuberculose: a experiência nacional. Cad Saúde Colet (Rio J.) 2010; 18:107-11.

19. Buchalla CM, Laurenti R, Ribeiro AF, Nitrini DR, Guarnieri CE, Carnauba EL, et al. Avaliação do uso da Classificação Internacional de Doenças para codificar a síndrome da imunodeficiência adquirida. Rev Saúde Pública 1996; 30:479-82.

20. Chiang CY, Lee MC, Yu MC, Enarson DA, Lin TP, Luh KT. Tuberculosis outcomes in Taipei: factors associated with treatment interruption for 2 months and death. Int J Tuberc Lung Dis 2009; 13:105-11.

21. Low S, Ang LW, Cutter J, James L, Chee CBE, Wang YT, et al. Mortality among tuberculosis patients on treatment in Singapore. Int J Tuberc Lung Dis 2009; 13:328-34.

22. Lindoso AABP, Waldman EA, Komatsu NK, Figueiredo SM, Taniguchi M, Rodrigues LC. Perfil de pacientes que evoluem para o óbito por tuberculose no Município de São Paulo, 2002. Rev Saúde Pública $2008 ; 42: 805-12$.

23. Albuquerque MFPM, Batista JA, Ximenes RAA, Carvalho MS, Diniz GTN. Risk factors associated with death in patients who initiate treatment for tuberculosis after two different follow-up periods. Rev Bras Epidemiol 2009; 12:513-22.

24. Malhão TA, Oliveira GP, Codenotti SB, Moherdaui F. Avaliação da completitude do Sistema de Informação de Agravos de Notificação da tuberculose, Brasil, 2001-2006. Epidemiol Serv Saúde 2010; 19:245-56.

25. Pacheco AG, Saraceni V, Tuboi SH, Moulton LH, Chaisson RE, Cavalcante SC, et al. Validation of a hierarchical deterministic record-linkage algorithm using data from 2 different cohorts of human immunodeficiency virus-infected persons and mortality databases in Brazil. Am J Epidemiol 2008; 168:1326-32.

26. Coutinho ES, Coeli CM. Acurácia da metodologia de relacionamento probabilístico de registros para identificação de óbitos em estudos de sobrevida. Cad Saúde Pública 2006; 22:2249-52.

27. Fonseca MG, Coeli CM, Lucena FFA, Veloso VG, Carvalho MS. Accuracy of a probabilistic record linkage strategy applied to identify deaths among cases reported to the Brazilian AIDS surveillance database. Cad Saúde Pública 2010; 26:1431-8.

28. Secretaria de Vigilância em Saúde, Ministério da Saúde. Especial tuberculose. Boletim Epidemiológico 2012; 43:1-12.

29. Medeiros D, Sucupira ED, Guedes RM, Costa AJL Análise da qualidade das informações sobre tuberculose no Município de Belford Roxo, Rio de Janeiro, 2006 a 2008. Cad Saúde Colet (Rio J.) 2012; 20:146-52.

Recebido em 17/Jun/2013

Versão final reapresentada em 25/Fev/2014

Aprovado em 25/Abr/2014 